Check for updates

Cite this: Mater. Horiz., 2019, 6, 176

Received 15th June 2018 Accepted 31st July 2018

DOI: $10.1039 / \mathrm{c} 8 \mathrm{mh} 00708 \mathrm{j}$

rsc.li/materials-horizons

Black phosphorus (BP) sponges synthesized electrochemically from bulk BP crystals comprise ultrathin (less than $4.0 \mathrm{~nm}$ ) and large (over tens of micrometers) BP nanosheets as the basic unit and the semi-connected nanosheets provide open channels. The general oxidation of BP can be prevented by well-chosen apparatus with tetrabutylphosphonium bromide as the electrolyte. The all-solid-state supercapacitor constructed with BP sponge as the electrode material exhibits a high capacitance of $80 \mathrm{~F} \mathrm{~g}^{-1}$ at $10 \mathrm{mV} \mathrm{s}{ }^{-1}$, which is much higher than those of bulk BP crystals and BP nanosheets. The BP sponges thus have great potential in energy storage applications. Furthermore, the modified electrochemical method provides the possibility for the high-quality exfoliation of other two-dimensional layered crystals to three-dimensional architectures.

Since the successful exfoliation of graphene in 2004, twodimensional (2D) layered materials with unique structural properties have attracted enormous attention especially in energy storage and conversion. ${ }^{1,2}$ However, the limited number of active sites, poor ion transport among restacked nanosheets, and blocked electron transport in the electrode materials have severely impaired the performance. ${ }^{3}$ Construction of a three-dimensional (3D) architecture using 2D nanosheets as the building block has been demonstrated to be an efficient way to exploit the impressive properties of $2 \mathrm{D}$ materials. ${ }^{4-8}$ First of all, by inhibiting restacking of 2D nanosheets with a large surface-to-volume ratio, the 3D architecture provides a large number of exposed active sites for ion absorption and reaction. Secondly, such a 3D architecture often provides pores or channels galore to facilitate ion diffusion and transport. Thirdly, the interpenetrating 2D nanosheets in the 3D structure

\footnotetext{
${ }^{a}$ Center for Biomedical Materials and Interfaces, Shenzhen Institutes of Advanced Technology, Chinese Academy of Sciences, Shenzhen 518055, P. R. China. E-mail:xf.yu@siat.ac.cn

${ }^{b}$ Department of Physics and Department of Materials Science and Engineering,

City University of Hong Kong, Tat Chee Avenue, Kowloon, Hong Kong, P. R. China $\dagger$ Electronic supplementary information (ESI) available. See DOI: 10.1039/ c8mh00708j

\$ Min Wen and Danni Liu contributed equally to this work.
}

\section{Conceptual insights}

Black phosphorus (BP) has attracted much research interest owing to its unique puckered two-dimensional (2D) structure, large surface area, high carrier mobility and strong mechanical strength, especially in energy storage and conversion applications. Construction of a three-dimensional (3D) structure using BP nanosheets as the building block might be an efficient way to exploit its impressive properties. However, the construction of 3D BP is very challenging because of its sensitivity to water, air and oxidants. In this work, a high-quality 3D BP sponge is synthesized in 3 minutes under ambient atmosphere by using a modified electrochemical approach. The BP sponges comprise ultrathin (less than $4.0 \mathrm{~nm}$ ), large (over tens of micrometers), and high-quality (unoxidized) BP nanosheets as the basic unit. The semi-connected nanosheets in the BP sponges provide plenty of open channels, and the all-solid-state supercapacitor constructed with the BP sponge as the electrode material has a high capacitance.

form an interconnected scaffold providing abundant paths for electron transport. Since the transport kinetics of ions and electrons among the materials are key to efficient energy storage and conversion, the design and construction of $3 \mathrm{D}$ structures from 2D materials is of scientific, technological, and commercial significance.

As an emerging 2D material, black phosphorus (BP) has attracted much research interest in physics, chemistry, and materials science. ${ }^{9-18}$ Owing to the unique puckered $2 \mathrm{D}$ structure, BP has a large surface area, high carrier mobility $\left(\approx 10000 \mathrm{~cm}^{2} \mathrm{~V}^{-1} \mathrm{~s}^{-1}\right)$, and strong mechanical strength $(\approx 94 \mathrm{GPa})$ which are favorable properties in energy storage and conversion applications. ${ }^{19-26}$ For instance, in lithium-ion and sodium-ion batteries, BP has a theoretical specific capacity of $2596 \mathrm{~mA} \mathrm{~h} \mathrm{~g}^{-1}$ which is about 7 times that of graphite. ${ }^{27}$ As an anode in sodium-ion batteries, BP nanosheets have been demonstrated to yield a specific capacity of $1968 \mathrm{~mA} \mathrm{~h} \mathrm{~g}^{-1}$ at $100 \mathrm{~mA} \mathrm{~g}^{-1}$ and the BP nanosheet-based supercapacitor shows a capacitance of $45.8 \mathrm{~F} \mathrm{~g}^{-1}$ at $10 \mathrm{mV} \mathrm{s}^{-1} \cdot{ }^{28,29}$ Since the 3D architecture of 2D nanomaterials is expected to deliver even better performance, the development of a 3D BP architecture is highly desired. In recent years, several 
strategies such as hydrothermal synthesis, ${ }^{30}$ chemical vapor deposition, ${ }^{31}$ template-assisted synthesis, ${ }^{32}$ and electrospinning ${ }^{33}$ have been proposed to convert 2D nanomaterials into 3D ones. However, in spite of recent advances, construction of a 3D BP structure is very challenging because of the sensitivity of BP to water, air and oxidants. ${ }^{34,35}$

In this work, a semi-connected 3D sponge comprising ultrathin BP nanosheets is synthesized by using a modified electrochemical approach, in which a special apparatus cooperating with a well-chosen bromide electrolyte ion is involved to prevent BP oxidation. Composed of ultrathin (less than $4.0 \mathrm{~nm}$ ), large (over tens of micrometers), and high-quality BP nanosheets as the basic unit, the BP sponges have plenty of channels boding well for electrochemical energy storage. The all-solid-state supercapacitor composed of BP sponge electrodes has a high specific capacitance of $80 \mathrm{~F} \mathrm{~g}^{-1}$ at $10 \mathrm{mV} \mathrm{s}^{-1}$ as well as excellent stability.

The BP sponges are synthesized using a two-electrode system under ambient conditions with bulk BP crystal as the cathode, Pt sheet as the counter electrode, and tetrabutylphosphonium bromide dissolved in $\mathrm{N}, \mathrm{N}$-dimethylformamide (DMF) as the electrolyte (Fig. 1a) (see details in Material and methods section). The electrochemical circuit is connected in a $\mathrm{H}$-type electrolytic cell with a cation-exchange membrane in the middle of the bridge. In this way, the bulk BP cathode can be separated with the Pt anode while the tetrabutylphosphonium cations can diffuse between the two electrodes. Furthermore, with bromine as the counter ion of quaternary phosphonium salt, the generated oxidation product cannot react with $\mathrm{BP}$ units in the synthesis process. The BP sponge is synthesized at a constant applied bias voltage of $-5 \mathrm{~V}$ and as shown in Fig. $1 \mathrm{~b}$, the bulk BP crystal expands rapidly by $\sim 90$ times after only 3 min. It is observed that the obtianed BP structure has a sponge-like morphology. This result might be related to the specific electrolyte. On the one hand, there are no gas bubbles for intercalation during the synthesis, so the BP sponge can maintain its morphology rather than separate into small pieces. Furthermore, since the diameter of tetrabutylphosphonium $(0.884 \mathrm{~nm})$ is much larger than the interplanar spacing of BP $(0.53 \mathrm{~nm}),{ }^{36-38}$ the efficient intercalation of cations promotes the fabrication of the sponge.

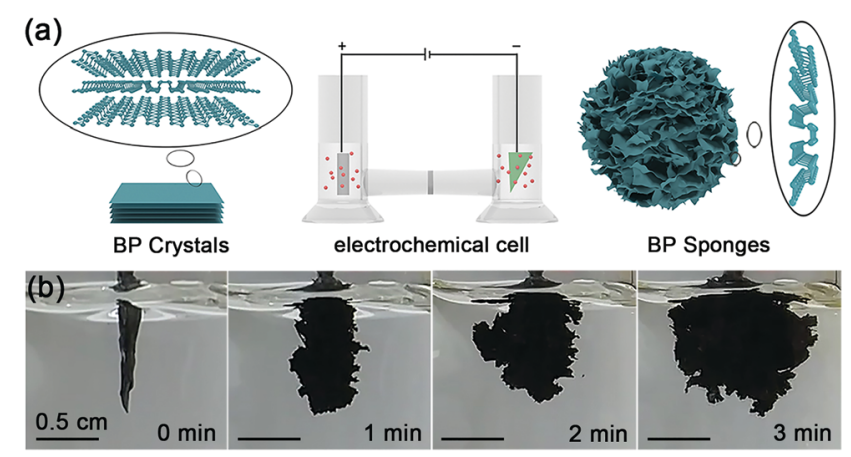

Fig. 1 Synthesis of BP sponge: (a) schematic illustration of bulk BP, the electrochemical cell, and BP sponge; (b) photographs taken at different times during synthesis.
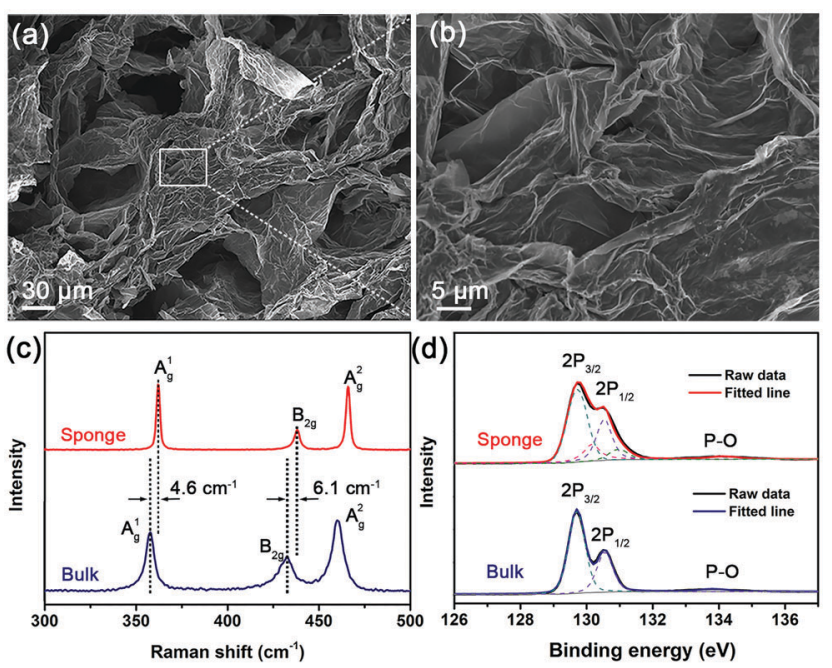

Fig. 2 Characterization of BP sponge: ( $a$ and b) SEM images; (c) Raman scattering and (d) XPS spectra (P 2p) of the BP sponge and bulk BP.

The as-generated BP sponge is characterized by scanning electron microscopy (SEM), Raman scattering, and X-ray photoelectron spectroscopy (XPS). As shown in Fig. 2a and b, the BP sponge consists of large BP nanosheets reaching over dozens of micrometers. The crumpled and layered BP units are quite different from the heavily stacked platelets in bulk BP (Fig. S1, ESI $\dagger$ ). The magnified SEM images in Fig. S2 (ESI $\dagger$ ) reveal that the preserved nanosheets in BP sponge are ultrathin and large. Furthermore, the semi-crosslinked 3D sponge possesses a lot of pores and small channels among the crumpled BP nanosheets. The large intrinsic BP nanosheets and the formed pores/channels are favorable to energy storage.

The Raman scattering spectra of the bulk BP and BP sponge are compared. As shown in Fig. 2c, the BP sponge exhibits three prominent peaks at $362.2,438.3$ and $466.1 \mathrm{~cm}^{-1}$ which are attributed to the $\mathrm{A}_{\mathrm{g}}^{1}, \mathrm{~B}_{2 \mathrm{~g}}$ and $\mathrm{A}_{\mathrm{g}}^{2}$ phonon modes, respectively. ${ }^{39}$ The in-plane and out-of-plane vibration modes indicate good crystalline quality. ${ }^{40}$ Compared to bulk BP, the vibrational modes blue-shift suggests reduced thickness and the presence of few-layer BP nanosheets in the sponge. ${ }^{41,42}$

The composition and chemical states of bulk BP and BP sponge are determined by XPS and the high-resolution $\mathrm{P} 2 \mathrm{p}$ and $\mathrm{O}$ 1s spectra are displayed in Fig. 2d, Fig. S3 and S4 (ESI $\dagger$ ), respectively. Obviously, the $\mathrm{BP}$ sponge exhibits characteristic doublets of $2 \mathrm{p}_{1 / 2}$ at $130.53 \mathrm{eV}$ and $2 \mathrm{p}_{3 / 2}$ at $129.72 \mathrm{eV}$, which are in accordance with those of bulk BP (130.54 eV and $129.71 \mathrm{eV})$. The small peaks at $130.95 \mathrm{eV}$ and $130.15 \mathrm{eV}$ might be ascribed to the changed $\mathrm{P}$ atoms at the edge of the nanosheets or the residual tetrabutylphosphonium electrolyte. ${ }^{43}$ Furthermore, compared with bulk BP, BP sponge exhibits similar O 1s spectra (Fig. S4, ESI $\dagger$ ) and negligible characteristic broad peak of phosphorus oxide (Fig. 2d and Fig. S3, ESI $\dagger$ ). This demonstrates that the BP sponge is not oxidized during the synthesis process. ${ }^{44}$ Even though oxidation often occurs during the exfoliation of BP nanosheets, it does not happen here because of the H-type electrolytic cell and the well-chosen bromide electrolyte used in 

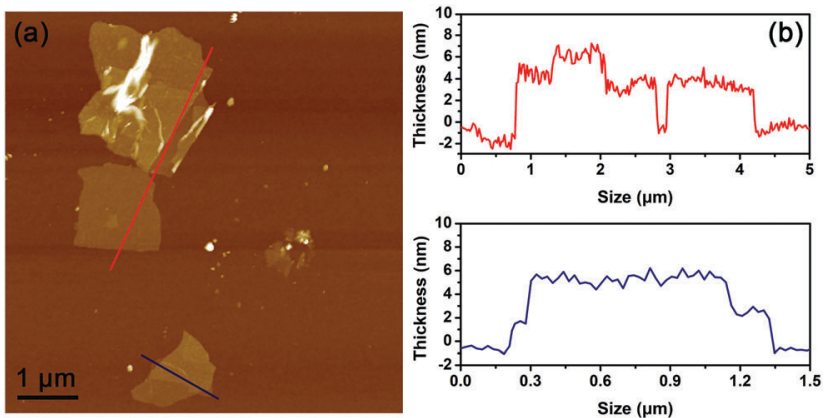

Fig. 3 AFM image of nanosheets in BP sponge and their height profiles.

the synthesis. Being placed on either side of the H-type cell, the bulk BP and Pt sheet are separated by a cation-exchange membrane. As the reaction proceeds, the solution in the anode gradually becomes orange due to the generated oxidation product (bromine) while the cathodic solution retains the original color (Fig. S5, ESI $\dagger$ ). With the cation-exchange membrane as the separator, oxidizing bromine generated at the anode is kept away from the phosphorus source and consequently, oxidation of BP is mitigated.

Atomic force microscopy (AFM), transmission electron microscopy (TEM) and selected area electron diffraction (SAED) are employed to further explore the basic unit in the semiconnected BP sponge. The separated 2D BP samples are obtained by sonicating small pieces of BP sponge in ethanol for $20 \mathrm{~min}$. The thickness of the BP nanosheets is investigated by AFM. As shown in Fig. 3a, the AFM image exihibits ultrathin BP nanosheets. From the curly and crumpled flakes, the thickness of BP ranges from 1.8 to $4.0 \mathrm{~nm}$, which suggests that the BP sponge is composed by few-layer BP nanosheets (Fig. 3b).

The thickness, morphology and crystal structure of the BP units are further examined by TEM and the coresponding SAED. ${ }^{45-47}$ Fig. 4a shows a typical ultrathin and large nanosheet with obvious wrinkles. More TEM images are shown in Fig. S6 (ESI $\dagger$ ) and the corresponding SAED patterns are displayed in Fig. 4b and Fig. S7 (ESI $\dagger$ ). The ultrathin BP is revealed by forbidden reflections such as (010) and (110). As shown by the simulated SAED patterns (Fig. S8, ESI $\dagger$ ), the (010) reflection indicates single-layer BP and the high-intensity (110) reflection confirms the presence of single-layer BP nanosheets. ${ }^{45}$ The high-resolution TEM images of the BP nanosheets are in good agreement with the above results. Fig. $4 \mathrm{c}$ and $\mathrm{d}$ show lattice fringes of $0.28,0.34$, and $0.44 \mathrm{~nm}$. This atomic arrangement is very different from that of the orthometric structure of multilayered BP but matches that of single-layer BP (Fig. 4e and f). ${ }^{47}$ These results show that the semi-connected BP sponge is composed of ultrathin BP nanosheets, among which some single-layer BP nanosheets exist.

Since the BP sponges contain ultrathin and large BP nanosheets and plenty of channels, good energy storage properties are expected. For verification and demonstration, an all-solid-state supercapacitor composed of the BP sponge electrode (BP-ASSP) is prepared as shown in Fig. 5a. In brief, the BP sponge is cut into small pieces and suspended in ethanol. After spreading on
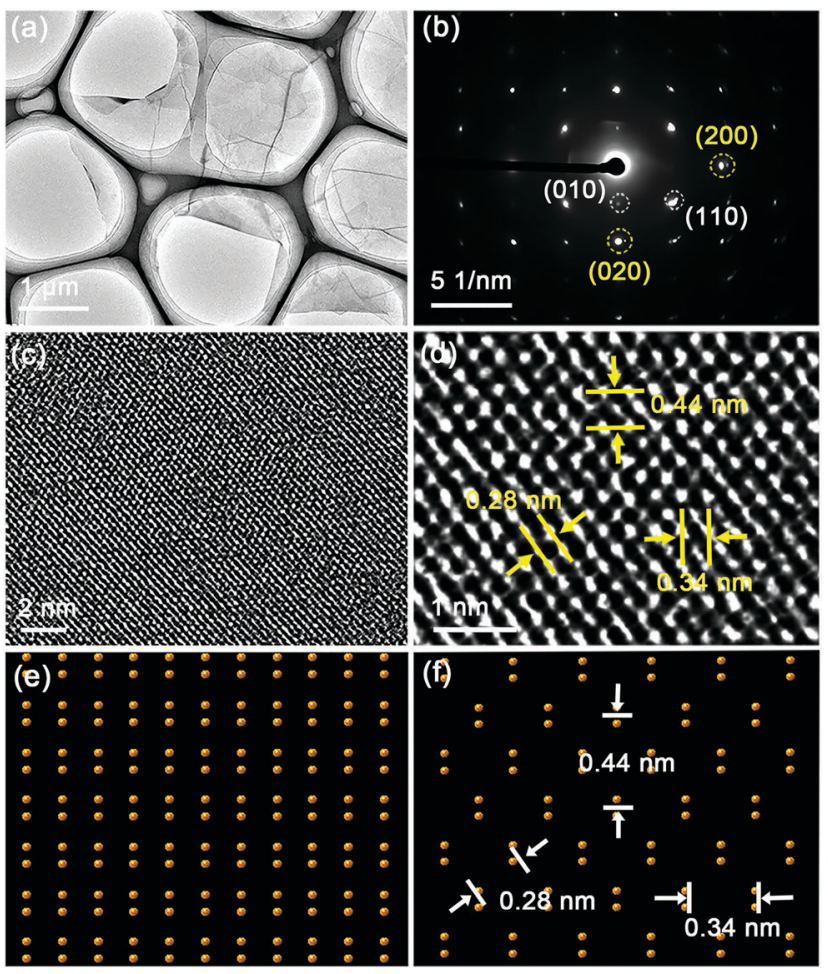

Fig. 4 Characterization of the nanosheets in BP sponge: (a) TEM image; (b) SAED pattern; (c) HR-TEM image; (d) magnified HR-TEM image; schematic atomic structures of (e) multilayer and (f) single-layer BP.

(a)
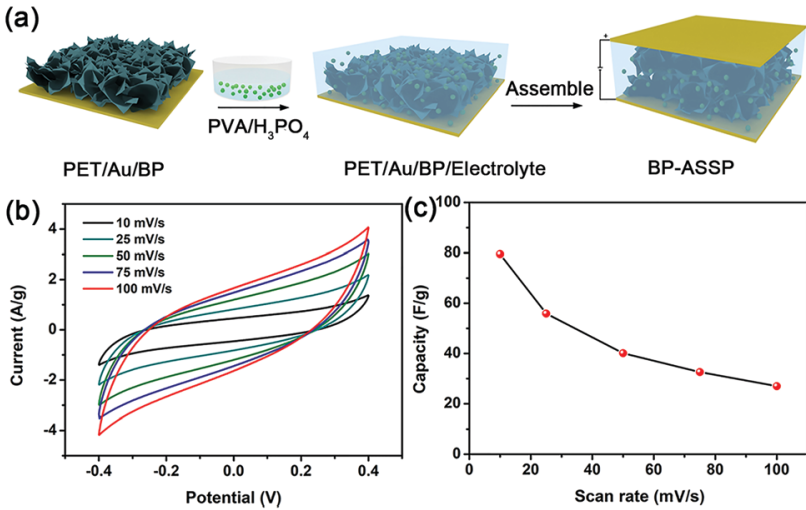

PET/Au/BP/Electrolyte

BP-ASSP
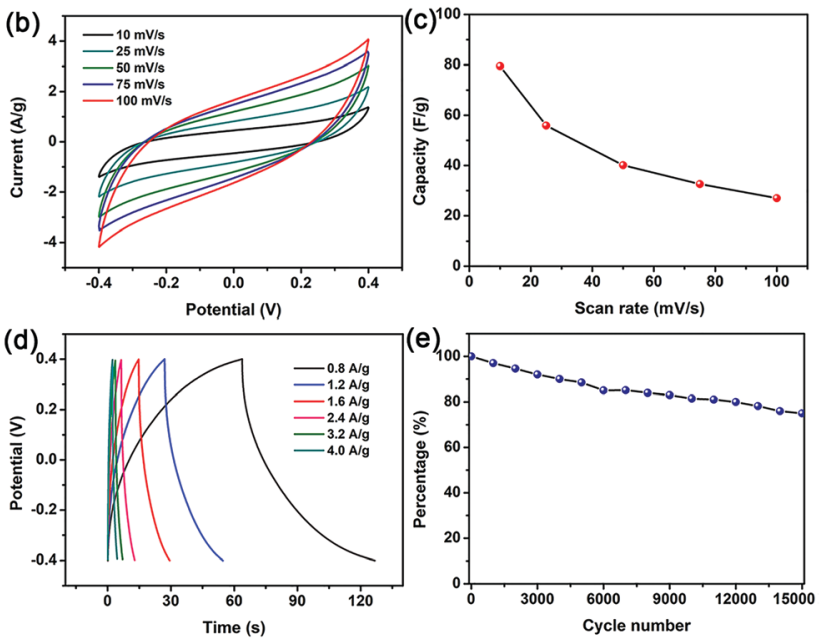

Fig. 5 Synthesis and performance of the BP-ASSP: (a) schematic illustration of the fabrication process; (b) cyclic voltammograms acquired at different scanning rates; (c) mass capacitances calculated from the CV curves at different scanning rates; (d) galvanostatic charging/ discharging curves obtained at different current densities; (e) cycle stability for 15000 cycles at a scanning rate of $100 \mathrm{mV} \mathrm{s}^{-1}$. 
the Au-coated PET substrate, the BP sponge electrodes are infiltrated with a gel electrolyte of poly(vinylalcohol)/phosphoric acid $\left(\mathrm{PVA} / \mathrm{H}_{3} \mathrm{PO}_{4}\right) .{ }^{48}$ The two electrodes are pressed together to fabricate a sandwiched all-solid-state supercapacitor. The assembly is carried out under ambient conditions (see details in Material and methods section). From the cross-section SEM image of the electrode, the thickness of BP is found to be $\sim 4 \mu \mathrm{m}$ (Fig. S9, ESI $\dagger$ ). And the amount of BP in the electrode is estimated to be $50 \mu \mathrm{g} \mathrm{cm} \mathrm{cm}^{-2}$ by using elemental analysis.

The performance of BP-ASSP is evaluated by cyclic voltammetry (CV). As shown in Fig. 5b, the CV curves at scanning rates between 10 and $100 \mathrm{mV} \mathrm{s}^{-1}$ show characteristic rectangular electrochemical double-layer capacitance curves. The corresponding mass capacitances of BP-ASSP at the different scanning rates are given in Fig. $5 \mathrm{c}$. At a scanning rate of $10 \mathrm{mV} \mathrm{s}^{-1}$, the BP-ASSP has a specific capacitance of $80 \mathrm{~F} \mathrm{~g}^{-1}$. Although the calculated capacitance decreases as the scanning rate increases, a high capacitance of $28 \mathrm{~F} \mathrm{~g}^{-1}$ is still observed at $100 \mathrm{mV} \mathrm{s}^{-1}$.

The galvanostatic charging/discharging (GCD) curves of the BP-ASSP at various current densities are presented in Fig. 5d. The GCD curves show typical electrochemical double-layer capacitance characteristics with a triangular shape indicating the good charge diffusion at the $\mathrm{BP}$ nanosheet $/ \mathrm{H}_{3} \mathrm{PO}_{4}$ interfaces during adsorption/desorption. The cycling ability is crucial in practice for supercapacitors and the BP-ASSP is assessed at a high scanning rate of $100 \mathrm{mV} \mathrm{s}^{-1}$. The specific capacitance retention is $80 \%$ after 15000 charging/discharging cycles confirming the good reversibility (Fig. 5e).

The excellent performance of BP sponge in the all-solid-state supercapacitor is possibly due to the following factors. Firstly, in the BP sponge, the ultrathin nanosheets provide a large ratio of exposed atoms and high carrier mobility. Secondly, the basic BP unit in the sponge is of high-quality and has a large size up to a few tens of micrometers thus facilitating the interfacial electron transfer kinetics since the high energy barriers among the individual nanosheets are remitted. Thirdly, the channels and pores in the BP sponge benefit electrolytic diffusion and promote ion transport. In contrast, the all-solid-state supercapacitors comprising bulk BP or 2D BP nanosheets as the electrode materials have much lower mass current densities (Fig. S10 and S11, ESI $\dagger$ ) further illustrating the superiority of the BP sponge.

\section{Conclusions}

In conclusion, 3D semi-connected BP sponges are synthesized by a rapid and simple electrochemical technique in $3 \mathrm{~min}$ under ambient atmosphere. The as-generated sponge consists of large (over tens of micrometers) and ultrathin (less than $4.0 \mathrm{~nm}$ ) BP nanosheets and the semi-connected nanosheets provide plenty of open channels. Although BP is very reactive, oxidation does not occur during synthesis due to the special apparatus and the well-chosen bromide electrolyte ion. As an electrode material in the all-solid-state supercapacitor, the BP sponge has a specific capacitance of $80 \mathrm{~F} \mathrm{~g}^{-1}$ at $10 \mathrm{mV} \mathrm{s}^{-1}$ which is much higher than those of bulk BP and 2D BP nanosheets.
The high-quality BP sponges have great potential in energy storage applications and will accelerate the development of BP-based technologies. In addition, the modified electrochemical method provides a possibility for the exfoliation of other two-dimensional layered crystals to three-dimensional architectures.

\section{Materials and methods}

\section{Synthesis of BP sponges}

The BP sponges were synthesized by a simple electrochemical method. It was performed using an IT6123B direct-current power supply in an H-type electrolytic cell with the bulk BP crystal as the cathode, platinum plate as the anode, and DMF containing $0.05 \mathrm{M}$ tetrabutylphosphonium bromide as the electrolyte. The cathode and anode were separated by a cation-exchange membrane. The cell voltage was $5 \mathrm{~V}$ and after $3 \mathrm{~min}$, the synthesized BP sponges were taken out and rinsed with DMF, acetone, $n$-hexane, and ethanol several times to remove impurities.

\section{Preparation of the $\mathrm{PVA} / \mathrm{H}_{3} \mathrm{PO}_{4}$ electrolyte}

PVA (1 g) was dispersed in deionized water $(10 \mathrm{~mL})$ and heated to $90{ }^{\circ} \mathrm{C}$ under continuous stirring until a clear solution was obtained. During the cooling process, $2 \mathrm{~g}$ of analytical reagent $\mathrm{H}_{3} \mathrm{PO}_{4}$ was gradually dropped into the solution under stirring and the gel electrolyte was a colorless and clear solution.

\section{Preparation of all-solid-state supercapacitor devices}

A PET substrate coated with $60 \mathrm{~nm}$ Au served as the current collector. Copper sheets were adhered to the edge of the Au-coated PET with silver plastic. A BP sponge was cut into small pieces and dispersed in ethanol. The suspension was spread on the Au-coated PET substrate and after evaporation of ethanol, the BP sponge electrode was covered by the $\mathrm{PVA} / \mathrm{H}_{3} \mathrm{PO}_{4}$ electrolyte with a dosage of $20 \mu \mathrm{L} \mathrm{cm}{ }^{-2}$. The $\mathrm{PVA} / \mathrm{H}_{3} \mathrm{PO}_{4}$ coated electrode was left under ambient conditions for infiltration. The excess water was removed by freeze drying. Two pieces of the $\mathrm{BP}$ sponge electrode were pressed together to form the sandwich device. After being encapsulated in epoxy resin, the device was used for further electrochemical measurements.

\section{Calculation of capacitance}

The specific capacitance of the device was calculated from the CV curves with the following eqn (1):

$$
C_{\mathrm{m}}=\int i(V) \mathrm{d} V / m \nu \Delta V
$$

where $C_{\mathrm{m}}$ refers to the mass capacitance of the device, $m$ is the mass of the electrode material, $\nu$ is the scanning rate, and $\Delta V$ is the potential window $(\mathrm{V})$.

\section{Conflicts of interest}

There are no conflicts to declare. 


\section{Acknowledgements}

This work was financially supported by the National Natural Science Foundation of China (51672305), Key Research Program of Frontier Sciences, CAS (QYZDB-SSW-SLH034), Leading Talents of Guangdong Province Program (00201520), and Hong Kong Research Grants Council (RGC) General Research Funds (GRF) (11301215 and 11205617).

\section{References}

1 C. Tan, X. Cao, X. J. Wu, Q. He, J. Yang, X. Zhang, J. Chen, W. Zhao, S. Han, G. H. Nam, M. Sindoro and H. Zhang, Chem. Rev., 2017, 117, 6225.

2 M. Chen, R. C. Haddon, R. Yan and E. Bekyarova, Mater. Horiz., 2017, 4, 1054.

3 L. Cong, H. Xie and J. Li, Adv. Energy Mater., 2017, 7, 1601906.

4 Q. Yun, Q. Lu, X. Zhang, C. Tan and H. Zhang, Angew. Chem., Int. Ed., 2018, 57, 626.

5 H. Wang, X. B. Li, L. Gao, H. L. Wu, J. Yang, L. Cai, T. B. Ma, C. H. Tung, L. Z. Wu and G. Yu, Angew. Chem., Int. Ed., 2018, 57, 192.

6 B. G. Choi, M. Yang, W. H. Hong, J. W. Choi and Y. S. Huh, ACS Nano, 2012, 6, 4020.

7 J. Zhang, Z. Li and X. W. D. Lou, Angew. Chem., Int. Ed., 2017, 56, 14107.

8 Q. Zhang, Y. Wang, B. Zhang, K. Zhao, P. He and B. Huang, Carbon, 2018, 127, 449.

9 H. Liu, A. T. Neal, Z. Zhu, Z. Luo, X. Xu, D. Tománek and P. D. Ye, ACS Nano, 2014, 8, 4033.

10 Y. Zhou, M. Zhang, Z. Guo, L. Miao, S.-T. Han, Z. Wang, X. Zhang, H. Zhang and Z. Peng, Mater. Horiz., 2017, 4, 997.

11 H. Wang, X. Yang, W. Shao, S. Chen, J. Xie, X. Zhang, J. Wang and Y. Xie, J. Am. Chem. Soc., 2015, 137, 11376.

12 R. Gusmao, Z. Sofer and M. Pumera, Angew. Chem., Int. Ed., 2017, 56, 8052 .

13 M. Batmunkh, M. Bat-Erdene and J. G. Shapter, Adv. Mater., 2016, 28, 8586.

14 J. Li, C. Chen, S. Liu, J. Lu, W. P. Goh, H. Fang, Z. Qiu, B. Tian, Z. Chen, C. Yao, W. Liu, H. Yan, Y. Yu, D. Wang, Y. Wang, M. Lin, C. Su and J. Lu, Chem. Mater., 2018, 30, 2742.

15 S. T. Han, L. Hu, X. Wang, Y. Zhou, Y. J. Zeng, S. Ruan, C. Pan and Z. Peng, Adv. Sci., 2017, 4, 1600435.

16 L. Viti, A. Politano and M. S. Vitiello, APL Mater., 2017, 5, 035602.

17 H. Yuan, X. Liu, F. Afshinmanesh, W. Li, G. Xu, J. Sun, B. Lian, A. G. Curto, G. Ye, Y. Hikita, Z. Shen, S. C. Zhang, X. Chen, M. Brongersma, H. Y. Hwang and Y. Cui, Nat. Nanotechnol., 2015, 10, 707.

18 L. Viti, J. Hu, D. Coquillat, W. Knap, A. Tredicucci, A. Politano and M. S. Vitiello, Adv. Mater., 2015, 27, 5567.

19 S. Wu, K. S. Hui and K. N. Hui, Adv. Sci., 2018, 1700491.

20 J. Qiao, X. Kong, Z. X. Hu, F. Yang and W. Ji, Nat. Commun., 2014, 5, 4475 .
21 Q. Wei and X. Peng, Appl. Phys. Lett., 2014, 104, 251915.

22 X. Zhu, T. Zhang, Z. Sun, H. Chen, J. Guan, X. Chen, H. Ji, P. Du and S. Yang, Adv. Mater., 2017, 29, 1605776.

23 M. Zhu, Z. Sun, M. Fujitsuka and T. Majima, Angew. Chem., Int. Ed., 2017, 57, 2160.

24 A. Sajedi-Moghaddam, C. C. Mayorga-Martinez, Z. Sofer, D. Bouša, E. Saievar-Iranizad and M. Pumera, J. Phys. Chem. C, 2017, 121, 20532.

25 H. Xiao, Z. S. Wu, L. Chen, F. Zhou, S. Zheng, W. Ren, H. M. Cheng and X. Bao, ACS Nano, 2017, 11, 7284.

26 M. Zhu, X. Cai, M. Fujitsuka, J. Zhang and T. Majima, Angew. Chem., Int. Ed., 2017, 56, 2064.

27 J. Sun, G. Zheng, H. W. Lee, N. Liu, H. Wang, H. Yao, W. Yang and Y. Cui, Nano Lett., 2014, 14, 4573.

28 Z. Huang, H. Hou, Y. Zhang, C. Wang, X. Qiu and X. Ji, Adv. Mater., 2017, 29, 1702372.

29 C. Hao, B. Yang, F. Wen, J. Xiang, L. Li, W. Wang, Z. Zeng, B. Xu, Z. Zhao, Z. Liu and Y. Tian, Adv. Mater., 2016, 28, 3194.

30 F. Zhou, S. Xin, H. W. Liang, L. T. Song and S. H. Yu, Angew. Chem., Int. Ed., 2014, 53, 11552.

31 X. Wang, X. Gan, T. Hu, K. Fujisawa, Y. Lei, Z. Lin, B. Xu, Z. H. Huang, F. Kang, M. Terrones and R. Lv, Adv. Mater., 2017, 29, 1603617.

32 Y. Ito, Y. Tanabe, H. J. Qiu, K. Sugawara, S. Heguri, N. H. Tu, K. K. Huynh, T. Fujita, T. Takahashi, K. Tanigaki and M. Chen, Angew. Chem., Int. Ed., 2014, 53, 4822.

33 D. Kong, H. He, Q. Song, B. Wang, W. Lv, Q.-H. Yang and L. Zhi, Energy Environ. Sci., 2014, 7, 3320.

34 A. Favron, E. Gaufres, F. Fossard, A. L. Phaneuf-L'Heureux, N. Y. Tang, P. L. Levesque, A. Loiseau, R. Leonelli, S. Francoeur and R. Martel, Nat. Mater., 2015, 14, 826.

35 S. Sinha, Y. Takabayashi, H. Shinohara and R. Kitaura, $2 D$ Mater., 2016, 3, 035010.

36 S. Yang, K. Zhang, A. G. Ricciardulli, P. Zhang, Z. Liao, M. R. Lohe, E. Zschech, P. W. M. Blom, W. Pisula, K. Mullen and X. Feng, Angew. Chem., Int. Ed., 2018, 57, 4677.

37 D. Ekka and M. N. Roy, J. Phys. Chem. B, 2012, 116, 11687.

38 F. Geng, R. Ma, Y. Yamauchi and T. Sasaki, Chem. Commun., 2014, 50, 9977.

39 S. Zhang, J. Yang, R. Xu, F. Wang, W. Li, M. Ghufran, Y.-W. Zhang, Z. Yu, G. Zhang, Q. Qin and Y. Lu, ACS Nano, 2014, 8, 9590.

40 M. B. Erande, S. R. Suryawanshi, M. A. More and D. J. Late, Eur. J. Inorg. Chem., 2015, 3102.

41 M. B. Erande, M. S. Pawar and D. J. Late, ACS Appl. Mater. Interfaces, 2016, 8, 11548.

42 Z. Guo, H. Zhang, S. Lu, Z. Wang, S. Tang, J. Shao, Z. Sun, H. Xie, H. Wang, X.-F. Yu and P. K. Chu, Adv. Funct. Mater., 2015, 25, 6996.

43 L. Liang, J. Wang, W. Lin, B. G. Sumpter, V. Meunier and M. Pan, Nano Lett., 2014, 14, 6400.

44 A. Ambrosi, Z. Sofer and M. Pumera, Angew. Chem., Int. Ed., 2017, 56, 10443.

45 A. Castellanos-Gomez, L. Vicarelli, E. Prada, J. O. Island, K. L. Narasimha-Acharya, S. I. Blanter, D. J. Groenendijk, M. Buscema, 
G. A. Steele, J. V. Alvarez, H. W. Zandbergen, J. J. Palacios and H. S. J. van der Zant, 2D Mater., 2014, 1, 025001.

46 P. Yasaei, B. Kumar, T. Foroozan, C. Wang, M. Asadi, D. Tuschel, J. E. Indacochea, R. F. Klie and A. Salehi-Khojin, Adv. Mater., 2015, 27, 1887.
47 R. Hultgren, N. S. Gingrich and B. E. Warren, J. Chem. Phys., 1935, 3, 351.

48 M. Wang, D. Duong le, N. T. Mai, S. Kim, Y. Kim, H. Seo, Y. C. Kim, W. Jang, Y. Lee, J. Suhr and J. D. Nam, ACS Appl. Mater. Interfaces, 2015, 7, 1348. 